\title{
Chiral symmetry breaking and the Josephson current in a ballistic superconductor-quantum wire-superconductor junction
}

\author{
I.V. Krive ${ }^{1,2}$, L.Y. Gorelik ${ }^{1}$, R.I. Shekhter ${ }^{1}$, and M. Jonson ${ }^{1}$ \\ ${ }^{1}$ Department of Applied Physics, Chalmers University of Technology and Göteborg University \\ SE-41296 Göteborg, Sweden \\ ${ }^{2}$ B. Verkin Institute for Low Temperature Physics and Engineering of the National Academy of \\ Sciences of Ukraine, 47 Lenin Ave., Kharkov 61103, Ukraine \\ Email: krive@ilt.kharkov.ua
}

Received November 7, 2003, revised December 15, 2003

\begin{abstract}
We evaluate the Josephson current through a quasi-1D quantum wire coupled to bulk superconductors. It is shown that the interplay of Rashba spin-orbit interaction and Zeeman splitting results in the appearence of a Josephson current even in the absence of any phase difference between the superconductors. In a transparent junction $(D \simeq 1)$ at low temperatures this anomalous supercurrent $J_{a n}$ appears abruptly for a Zeeman splitting of the order of the Andreev level spacing as the magnetic field is varied. In a low transparency $(D<<1)$ junction one has $J_{a n} \propto \sqrt{D}$ under special (resonance) conditions. In the absence of Zeeman splitting the anomalous supercurrent disappears. We have investigated the influence of dispersion asymmetry induced by the Rashba interaction in quasi-1D quantum wires on the critical Josephson current and have shown that the breakdown of chiral symmetry enhances the supercurrent.
\end{abstract}

PACS: 74.80.Fp, 31.30.Gs, 71.70.Ej, 73.20.Dx

\section{Introduction}

Quantum wires (QWs) have the potential of being the basic elements in future nanometer-scale electronic devices. Electron transport in QWs is coherent and ballistic. It results in a number of spectacular phenomena such as conductance quantization, persistent current oscillations in ring-shaped wires (quantum rings), etc. At present, QWs are realized experimentally in the form of long and narrow channels ( $L \gg d \sim \lambda_{F}$, where $\lambda_{F}$ is the electron Fermi wavelength) in a two-dimensional electron gas (2DEG) (see Ref. 1) and as conducting 1D and quasi-1D molecular systems. The most successful and promising realization of $1 \mathrm{D}$ conductors is single-wall carbon nanotubes [2]. It has been shown both theoretically [3] and experimentally [4] that in carbon nanotubes the effects of Coulomb interaction are significant and that they transform the conduction electron system into a Luttinger liquid. In quantum wires formed in 2DEG the electron-electron interaction is less pronounced (presumably due the screening effects of the nearby bulk metallic electrodes) and electron transport in these systems can in many cases be successfully described by Fermi liquid theory.

It has long been known [5] that electrons confined to a plane (e.g., in MOSFET structures or in heterostructures) experience a strong spin-orbit interaction originating from interface electric field (Rashba spin-orbit interaction). Recently it was shown experimentally that the strength of the Rashba coupling can be controlled by a gate voltage [6]. The Rashba effect leads to various interesting suggestions in spintronics and it has been a subject of active theoretical and experimental studies in recent years (see, e.g., Ref. 7 and references therein).

For quasi-1D electron systems the influence of the Rashba interaction on thermodynamic and transport properties of quantum wires were considered in Refs. 8 and 9. There it was shown that in the presence of an in-plane confinement potential and spin-orbit interactions the electron spectrum is qualitatively 
modified. Chiral symmetry, which is usually assumed to be present in QWs, is violated, resulting in the appearence of a dispersion asymmetry. To be more precise, the right-moving spin-up* (left-moving spin-down) and left-moving spin-up ( right-moving spin-down) electrons have different Fermi velocities [8]. This implies that electrons in quantum wires with Rashba interaction are «chiral particles», and their spin projections are correlated with the direction of motion. Being interested in low-energy $\left(E<<\varepsilon_{F}\right)$ properties of quantum wires, we can classify these particles as belonging to two subbands ( $\ll 1 »$ and $\ll 2 »)$ characterized by their Fermi velocities (see Fig. 1). Notice that this electron spectrum pertains to a weak or moderate spin-orbit interaction. As was demonstrated in Ref. 10 for a strong Rashba interaction, the projection of electron spin is strongly correlated with the direction of motion, and left- and right-moving electrons with the Fermi energy always have opposite spin projections.

The unusual spectral properties of electrons in QWs have to show up in situations when spin degrees of freedom are nontrivially involved in the electron dynamics. Here we consider the Josephson current in a long $\mathrm{S}-\mathrm{QW}-\mathrm{S}$ junction for an electron spectrum with dispersion asymmetry and large Zeeman splitting. Recently the combined effect of Zeeman and spin-orbit interactions on the Josephson current in a short ballistic junction formed in a 2DEG was theoretically studied [11]. Notice that interaction induced dispersion asymmetry in the electron spectrum is a specific pro-

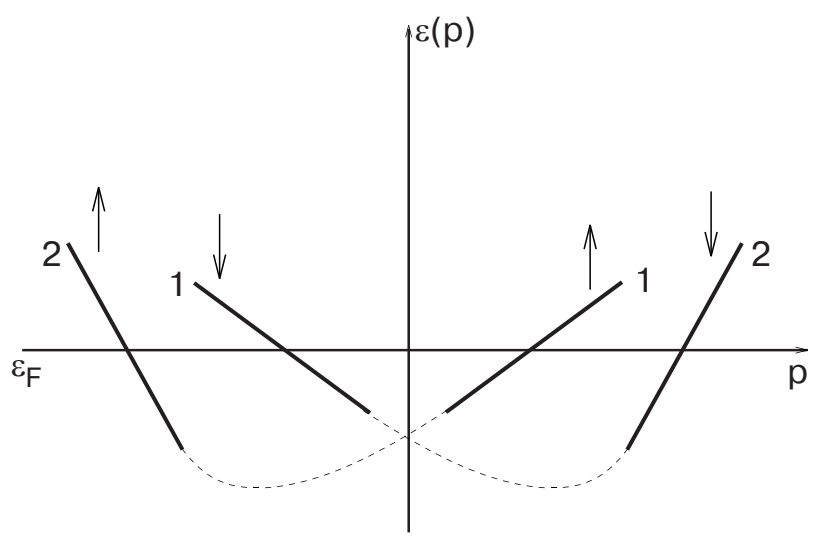

Fig. 1. Schematic energy spectrum of $1 \mathrm{D}$ electrons with dispersion asymmetry. The particles with energies close to the Fermi energy $\varepsilon_{F}$ have an almost linear dependence on momentum and are classified by their Fermi volocities $\left(v_{1 F}-\right.$ subband $1, v_{2 F}-$ subband 2$)$. perty of quasi-one-dimensional geometry [8]. For a pure 2D geometry spin-orbit interaction does not lead to a chiral symmetry breaking, and the peculiar effects produced by chiral particles do not manifest themselves in the short and wide SNS junction considered in Ref. 11. In particular, we show that the combined effect of Rashba and Zeeman interactions results in the appearence of an anomalous Josephson current $J_{a n}=J(\varphi=0)$ in a long $\mathrm{S}-\mathrm{QW}-\mathrm{S}$ junction. For a transparent junction the induced by Rashba and Zeeman interaction supercurrent at low temperatures is a step-like periodic or quasiperiodic function of magnetic field (see Fig. 2). The periodicity depends on the ratio of the Fermi velocities and is controlled by the strength of the spin-orbit interaction. The amplitude of the anomalous current at $T=0$ is of the order of the critical Josephson current in a long $\mathrm{S}-\mathrm{N}-\mathrm{S}$ junction and it appears abruptly at finite values of Zeeman splitting of the order of the Andreev level spacing (Fig. 2). For a low-transparency junction $(D<<1)$, realized by introducing a scattering barrier (impurity) into the normal region, the maximum amplitude of $J_{a n}$ at special (resonance) conditions is of the order of $\sqrt{D}$. Notice that this unusual dependence
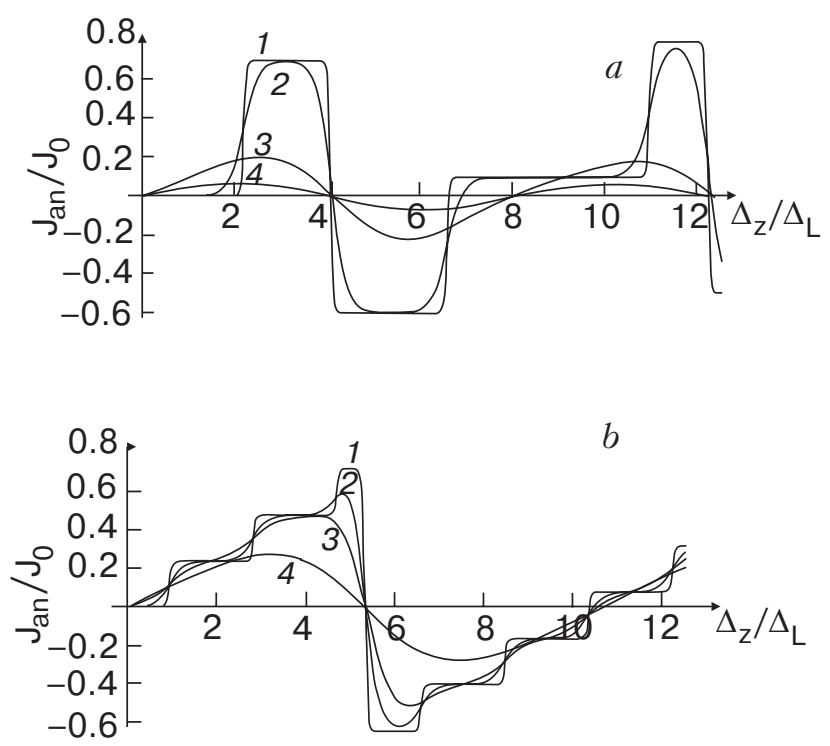

Fig. 2. The dependence of the normalized anomalous Josephson current $J_{a n} / J_{0}\left(J_{0}=e v_{F} / L\right)$ on the dimensionless Zeeman splitting $\Delta_{Z} / \Delta_{L}\left(\Delta_{L}=\hbar v_{F} / L\right)$. (a) asymmetry parameter $\lambda_{a}=0.3$. The different plots $(1-4)$ correspond to different temperatures $T=(0.1,0.5,3,5) T^{*}$ where $T^{*}=$ $=\Delta_{L} / 2 \pi$. (b) $\lambda_{a}=0.7 ; T=(0.1,0.5,1,3) T^{*}$.

* Under the conditions of Rashba effect the electron spin lies in the plane of the heterostructure transition layer and it is always perpendicular to the electron momentum. The terms spin-«up» and spin-«down» determine two opposite spin projections orthogonal to the line of the quantum wire at the $2 \mathrm{D}$ interface. 
on transparency, which corresponds to tunneling of a single electron through a barrier (its entangled partner resonantly passes through the structure) holds also for the critical Josephson current even in the absence of dispersion asymmetry. This effect can be interpreted as a tunnel splitting of the deGennes-Saint-James bound state [12] shifted by magnetic field to the vicinity of the Fermi level. Analogous effects of giant critical supercurrents in tunnel SIS («I» stands for insulator) and SINIS structures were discussed in Ref. 13 (see also Ref. 14, where resonance effects are considered for a persistent current in a normal metal ring).

When the Zeeman splitting vanishes, the anomalous supercurrent and all the above described resonance effects disappear. Then the Josephson current in a low-D junction is small $(\sim D)$. What is the influence of dispersion asymmetry on the critical current? There is a general statement [15] that spin-orbit interaction in systems with the Aharonov-Bohm geometry suppresses persistent currents. Although the theorem directly concerns normal ring-shaped conductors, it also holds for linear hybrid systems with Andreev mirrors due to the analogy between persistent currents in a normal 1D ring and Josephson currents in a long SNS junction. We show here that the cited statement is not valid when the spin-orbit interaction is accompanied by chiral symmetry breaking. Rashba spin-orbit interaction in quantum wires always enhances the critical current.

\section{Anomalous Josephson current}

The Josephson current, being an equilibrium supercurrent between two superconductors, can be calculated from the general thermodynamic relation

$$
J=\frac{e}{\hbar} \frac{\partial \Omega}{\partial \varphi}
$$

where $\Omega$ is the thermodynamic potential of the junction considered and $\varphi$ is the phase difference between the two superconductors. We have included a factor of 2, which usually appears in Eq. (1) in combination with the electric charge, into the definition of $\Omega$. This factor originates from spin degeneracy, and in the presence of Zeeman splitting $\Omega=\Omega_{\uparrow}+\Omega_{\downarrow}$ and $\Omega_{\uparrow} \neq \Omega_{\downarrow}$.

In general both the Andreev bound states $(E<|\Delta|$, $\Delta$ is the superconducting order parameter) and the continuum scattering states $(E \geq|\Delta|)$ contribute to the supercurrent. In two limiting cases - short ( $L<<\xi_{0}=\hbar v_{F} /|\Delta|, L$ is the junction length) and long $\left(L>>\xi_{0}\right)$ junctions - only bound states are relevant. This statement is well known for the case of short junctions (see, e.g., Ref. 16 and references therein). For a long junction it has been shown that the Josephson current through a long SNS junction does not depend on $|\Delta|$ at all [17]. Then, one can formally put $|\Delta| \rightarrow \infty$ and sum over all Andreev bound states $E_{n}(n=0, \pm 1, \pm 2, \ldots)$ with the natural assumption that the supercurrent vanishes in the limit $L \rightarrow \infty$. This procedure (analogous to Casimir energy evaluation in quantum field theory; see, e.g., Ref. 18) reproduces all known results for a long ballistic SNS junction. In what follows we will consider only long junctions.

To get analytical results we consider a single barrier junction of length $L$, where the barrier is located at the point $x=l \leq L$; the distance $l$ is measured from the left bank of the junction (see Fig. 3). We start with the general equation for Andreev bound state energies expressed in terms of scattering matrices of electrons $\left(\hat{S}_{E}\right)$ and holes $\left(\hat{S}_{-E}^{*}\right)$ in the normal region [19]

$$
\operatorname{det}\left(1-\alpha_{A} \hat{S}_{E} \hat{r}_{A} \hat{S}_{-E}^{*} \hat{r}_{A}^{*}\right)=0
$$

where $\alpha_{A}=\exp [-2 i \arccos (E /|\Delta|)] \simeq-1$ in our case $(E<<)$, and $\hat{r}_{A}$ is a diagonal matrix that only depends on the superconducting phases (see Appendix). Since the presence of a magnetic field violates T-symmetry, the two-channel scattering of spin- $1 / 2$ electrons is described by a $4 \times 4$ nonsymmetric unitary matrix $\hat{S}_{E}$. The normal backscattering in our approach (we neglect spin flips induced by a weak Rashba interaction) is always accompanied by a change of «channel index» $1 \Leftrightarrow 2$ (see Fig. 1). This allows us to parametrize the scattering matrix by 7 independent real parameters (see Appendix).

After straightforward (although rather lengthy) transformations, Eq. (2) results in the transcendental equation for Andreev level energies of the form

$$
\begin{aligned}
\cos [(E & \left.\left.+\frac{\eta}{2} \Delta_{Z}\right) \delta_{L}^{(+)}\right]+R \cos \left[\left(E+\frac{\eta}{2} \Delta_{Z}\right) \delta_{L-2 l}^{(+)}\right]+ \\
& +D \cos \left[\left(E+\frac{\eta}{2} \Delta_{Z}\right) \delta_{L}^{(-)}+\eta \varphi\right]=0
\end{aligned}
$$

Here $\Delta_{Z}=g \mu_{B} H$ is the Zeeman energy splitting ( $g=2$ for bare electrons, and we assume that the magnetic field $H$ is locally applied only on the quantum

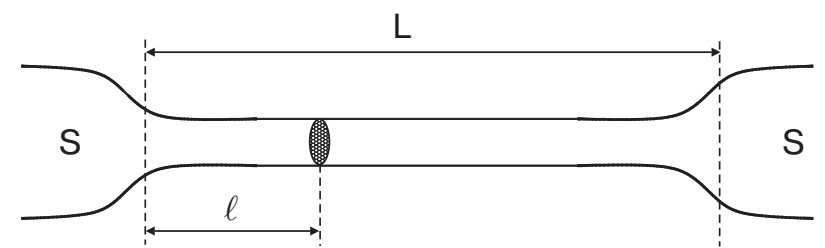

Fig. 3. A long $\left(L>>\xi_{0}\right)$ ballistic $\mathrm{S}-\mathrm{N}-\mathrm{S}$ junction with a scattering barrier (shaded region). 
wire), $\eta= \pm 1, D$ is the transparency of the junction $D+R=1$, and

$$
\delta_{x}^{( \pm)}=\frac{x}{\hbar}\left(\frac{1}{v_{1 F}} \pm \frac{1}{v_{2 F}}\right) .
$$

In the limit $H=0$ and $v_{1 F}=v_{2 F}$ (absence of spin-orbit interaction) Eq. (3) is reduced to a well-known spectral equation for Andreev levels in a long SNS junction with a single barrier $[16,20]$.

For a transparent junction $(D=1)$ the Andreev bound states are described by two independent sets of energy levels

$$
\begin{aligned}
& E_{n, \eta}^{(1)}=\pi \Delta_{L}^{(1)}\left(n+\frac{1}{2}+\eta \frac{\varphi+\chi_{1}}{2 \pi}\right), \\
& E_{m, \eta}^{(2)}=\pi \Delta_{L}^{(2)}\left(m+\frac{1}{2}+\eta \frac{\varphi-\chi_{2}}{2 \pi}\right),
\end{aligned}
$$

where the integers $n, m=0, \pm 1, \pm 2, \ldots$ and $\eta= \pm 1$ are the standard quantum numbers of the Andreev-Kulik spectrum in a perfectly transmitting SNS junction [21]; $\Delta_{L}^{(j)}=\hbar v_{j F} / L$ and $\chi_{j}=\Delta_{Z} / \Delta_{L}^{(j)}$ is the phase acquired due to Zeeman interaction. Notice that for a given band index $(\ll 1 »$ or $\ll 2 »)$ the relative sign between the superconducting phase difference $\varphi$ and the magnetic phase $\chi_{j}$ is fixed and is different for channels «1» and «2». This is a direct consequence of the chiral properties of the electrons in our model. In the absence of spectral asymmetry the two energy spectra Eq. (5) correspond to Zeeman-split Andreev levels.

Knowing explicitly the energy spectrum Eq. (5), it is straightforward to evaluate the Josephson current. It takes the form

$$
\begin{gathered}
J(\varphi, T, H)=\frac{2 e T}{\hbar} \times \\
\times \sum_{k=1}^{\infty}(-1)^{k+1}\left\{+\frac{\sin k\left(\varphi+\chi_{1}\right)}{\sinh \left(2 \pi k T / \Delta_{L}^{(1)}\right)}+\frac{\sin k\left(\varphi-\chi_{2}\right)}{\sinh \left(2 \pi k T / \Delta_{L}^{(2)}\right)}\right\} .
\end{gathered}
$$

It is readily seen from Eq. (6) that an anomalous Josephson current $J_{a n} \equiv J(\varphi=0)$ appears only if both the Zeeman splitting $\left(\Delta_{Z}\right)$ and dispersion asymmetry $\left(v_{1 F} \neq v_{2 F}\right)$ are nonzero. Here we use the term «anomalous» just to define the supercurrent at $\varphi=0$. Actually this current is induced by Zeeman splitting in a $1 \mathrm{D}$ electron system with dispersion asymmetry. The direction (sign) of $J_{a n}$ is totally determined by the sign of asymmetry parameter (see Eq. (8)). At high temperatures $T \geq \Delta_{L}^{(j)}$ the anomalous supercurrent is exponentially small. In the low-temperature region $\left(T \ll \Delta_{L}^{(j)}\right)$ it is a piecewise constant function of magnetic field represented by the series

$$
J_{a n}(H)=\frac{e}{\pi L} \sum_{k=1}^{\infty} \frac{(-1)^{k+1}}{k}\left(v_{1 F} \sin k \chi_{1}-v_{2 F} \sin k \chi_{2}\right) .
$$

For rational values $v_{1 F} / v_{2 F}=p / q(p \leq q$ are the integers) $J_{a n}$ is a periodic function of magnetic field with period $\delta H=2 \pi q \Delta_{L}^{(1)} / g \mu_{B}$; otherwise it is a quasiperiodic function.

It is convenient to introduce the mean Fermi velocity $v_{F}=\left(v_{1 F}+v_{2 F}\right) / 2$ and the asymmetry parameter $\lambda_{a}$,

$$
\lambda_{a}=\frac{v_{1 F}-v_{2 F}}{v_{1 F}+v_{2 F}},
$$

which determines the strength of Rashba spin-orbit interaction in a $1 \mathrm{D}$ quantum wire. The dependence of the normalized anomalous supercurrent $J_{a n} / J_{0}\left(J_{0}=\right.$ $\left.=e v_{F} / L\right)$ on the dimensionless Zeeman splitting $\chi=\Delta_{Z} / \Delta_{L}\left(\Delta_{L}=\hbar v_{F} / L\right)$ for $\lambda_{a}=0.3$ and for different temperatures is shown in Fig. 2, $a$. In the limit of strong asymmetry (this range of parameters, however, seems to be unrealistic[8] ), when only one of the two channels $(\ll 1 »$ or $\ll 2 »)$ contributes to Eq. (6), the dependence of the anomalous current on the magnetic field becomes analogous to the well-known phase dependence of the Josephson current [21]. The approach to this simple behavior passes via the stages of staircase-like dependences (see Fig. 2,b). Notice that we plotted the figures assuming that $v_{1 F} \geq v_{2 F}$. The interchange $v_{1 F} \leftrightarrow v_{2 F}$ makes the supercurrent Eq. (7) change sign.

\section{Giant critical current in a magnetically controlled low-transparency junction}

Now we consider the limit $D<<1$ pertaining to low-transparency SNS junctions. As is well known (see, e.g., Refs. 16,20), the supercurrent in this limit for a single barrier junction in the absence of Zeeman and spin-orbit interactions is described by the simple formula $J(\varphi)=J_{C} \sin \varphi$, where the critical current at low temperatures $T \ll \Delta_{L}$ is of the order of $D$ $\left(J_{c} \sim \operatorname{Dev}_{F} / L\right)$. Interesting physics for low- $D$ junctions appears when resonant electron tunneling occurs. This is, for instance, the case for the symmetric double-barrier ballistic junction considered in Refs. 13,16. There it was shown that for resonance conditions (realized for a special set of junction lengths) a giant critical supercurrent appears, $J_{c} \propto D_{b}$, where $D_{b}$ is the transparency of a single barrier. Analogous results were obtained for the persistent current in a ballistic ring with a double barrier [14]. Notice that for the symmetric structure considered in Ref. 13 the normal current (transmission coefficient, which determines the current through a double-barrier structure with normal 
leads) at resonance conditions does not depend on barrier transparency at all. This means that for the hybrid structure considered in Ref. 13, the superconductivity actually suppresses electron transport.

We show below that in a magnetically controlled single-barrier junction there are conditions when superconductivity in the leads strongly enhances electron transport, and a mesoscopic hybrid structure is characterized by a giant critical current $J_{c} \propto \sqrt{D}$

We start with the case of a symmetric single-barrier junction, when the scattering barrier is situated in the middle of the normal region, i.e., $l=L / 2$ in Eq. (3). Then $\delta_{x=0}^{( \pm)}=0$, and the second cosine term in the spectral equation Eq. (3) is equal to one:

$$
\cos \left[\left(E+\frac{\eta}{2} \Delta_{Z}\right) \delta_{L-2 l}^{(+)}\right]=1 .
$$

When the condition (9) is fulfilled, the spectral equation is reduced to the much simpler expression

$$
\begin{gathered}
\cos ^{2}\left[\frac{1}{2}\left(E+\frac{\eta}{2} \Delta_{Z}\right) \delta_{L}^{(+)}\right]= \\
=D \sin ^{2}\left[\frac{1}{2}\left(E+\frac{\eta}{2} \Delta_{Z}\right) \delta_{L}^{(-)}+\eta \varphi\right] .
\end{gathered}
$$

By using Eq. (10), one can readily evaluate the partial supercurrent $j_{\{\alpha\}}$ characterized by 3 quantum numbers $\{\alpha\}=(n, \eta, \sigma)$ where $n=0, \pm 1, \pm 2, \ldots ; \eta, \sigma= \pm 1$ :

$$
j_{\{\alpha\}}=\frac{e}{\hbar} \frac{\partial E_{\{\alpha\}}}{\partial \varphi}=-\frac{e \eta \sigma}{\hbar} \frac{\sqrt{D} \cos \frac{1}{2}\left[E_{\{\alpha\}} \delta_{L}^{(-)}+\eta\left(\frac{1}{2} \Delta_{Z} \delta_{L}^{(-)}+\varphi\right)\right]}{\delta_{L}^{(+)} \sin \frac{1}{2}\left[\frac{\delta_{L}^{(+)}}{2}\left(E_{\{\alpha\}}+\frac{\eta}{2} \Delta_{Z}\right)\right]+\sigma \delta_{L}^{(-)} \sqrt{D} \cos \frac{1}{2}\left[E_{\{\alpha} \delta_{L}^{(-)}+\eta\left(\frac{1}{2} \Delta_{Z} \delta_{L}^{(-)}+\varphi\right)\right]},
$$

where $E_{\{\alpha\}} \equiv E_{n, \eta, \sigma}$ is a solution of Eq. (10). The Josephson current at $T=0$ is a sum of partial currents over all occupied states.

The resonance current (of order $\sqrt{D}$ ) is formed by noncompensated partial currents carried by the Andreev levels in the vicinity of the Fermi energy, i.e., for $E_{\{\alpha\}}=0^{-}$when $D \rightarrow 0$. Such levels exist only for a discrete set of Zeeman splittings

$$
\Delta_{Z}^{(k)}=\frac{2 \pi(2 k+1)}{\delta_{L}^{(+)}}, \quad k=0,1,2, \ldots
$$

At a given $\Delta_{Z}^{(k)}$ (controlled, e.g., by an external local magnetic field) two $(\eta= \pm 1)$ Andreev levels contribute to the resonance Josephson current $J_{r}$, which can be represented in the form

$$
J_{r}(\varphi)=J_{0} \sqrt{D} \frac{1-\lambda_{a}^{2}}{2} \frac{\sin \left(\frac{1}{2} \Delta_{Z}^{(k)} \delta_{L}^{(+)} \lambda_{a}+\varphi\right)}{\left|\sin \frac{1}{2}\left(\frac{1}{2} \Delta_{Z}^{(k)} \delta_{L}^{(+)} \lambda_{a}+\varphi\right)\right|},
$$

where $J_{0}=e v_{F} / L$, and the asymmetry parameter $\lambda_{a}$ is defined in Eq. (8).

In the absence $\left(\lambda_{a}=0\right)$ of spin-orbit interaction Eq. (13) has the typical form of a resonance Josephson current (see, e.g., Ref. 16) associated with the contribution of a single Andreev level. One can interpret this result as follows. Let us assume for a moment that the potential barrier is infinite. Then, a symmetric
SNINS junction ( «I» stands for the insulator «layer») breaks into two identical INS-hybrid structures. In each of the two systems de Gennes-Saint-James energy levels [12] with spacing $2 \pi \hbar v_{F} / L$ are formed. For a finite barrier these levels are split with the characteristic splitting energy $\delta \sim \sqrt{D} \Delta_{L}<<\Delta_{L}$. The tunnel-split levels, being localized already on the whole length $L$ between the two superconductors, are nothing but the Andreev energy levels, i.e., they depend on the superconducting phase difference. Although the partial current of a single level is large (of the order of $\sqrt{D}$, see Ref. 13,16), the current carried by a pair of split levels is small $(\sim D)$ due to a partial cancellation. A Zeeman splitting of order $\Delta_{L}$ shifts the set of Andreev levels so that the Fermi energy lies in between the split levels. Now only the lower state is occupied, and this results in an uncompensated, large Josephson current. In other words only one of the two electrons of the Cooper pairs that form the supercurrent tunnels through the barrier. Its entangled partner at resonance conditions passes through the hybrid SFIFS structure $(\ll \mathrm{F} \gg$ denotes the region with nonzero Zeeman splitting) without backscattering. Since the quantized electron-hole spectrum is formed by Andreev scattering, the resonance structure for a single barrier junction disappears when the leads are in the normal state. Hence, electron transport through a normal region in our case is enhanced by superconductivity. 
The effect of chiral symmetry breaking on the physical picture described above is to additionally split the degenerate Andreev levels. A dispersion asymmetry $\lambda_{a} \neq 0$ lifts the left-right symmetry of electron transport through the junction and splits the doubly degenerate Andreev levels at $\varphi=0$. This results in appearence of giant anomalous Josephson current (see Eq. (13)) at $\varphi=0$.

We saw that it is indispensable that Eq. (9) holds in order for resonant transport through the single barrier hybrid structure to occur. This equation can be satisfied not only for the symmetric junction considered above. One can easily check that for a fixed value of Zeeman splitting $\Delta_{Z}^{(k)}$ given by Eq. (12) there is a set of points where a barrier still supports resonant transport. These points, determined by their coordinates $x_{m}^{(k)}$ measured from the middle of a junction, are ( $m$ is an integer)

$$
x_{m}^{(k)}= \pm \frac{m}{2 k+1} L, \quad 0 \leq m \leq k+1 / 2 .
$$

The temperature dependence of the $\sqrt{D}$-currents is determined by the energy scale $\delta \sim \sqrt{D} \Delta_{L}$, and at temperatures $\bar{T} \geq \delta$, which are much less then $\Delta_{L}$, all resonance effects are washed out.

\section{Influence of chiral symmetry breaking on the critical current}

There is a general statement [15] that spin-orbit interaction in 1D systems with the Aharonov-Bohm geometry produces additional reduction factors in the Fourier expansion of thermodynamic or transport quantities. This statement holds for spin-orbit Hamiltonians for which the transfer matrix is factorized into spin-orbit and spatial parts. In a pure 1D case the spin-orbit interaction is represented by the Hamiltonian $\mathcal{H}_{1 D}^{(s o)}=\alpha_{(s o)} \hat{p}_{x} \sigma_{z}$, which is the product of spin-dependent and spatial operators, and thus it satisfies the above-described requirements. However, as was shown by direct calculation in Ref. 8, spin-orbit interaction of electrons in 1D quantum wires formed in 2DEG by an in-plane confinement potential cannot be reduced to the Hamiltonian $\mathcal{H}_{1 D}^{(s o)}$. Instead, a violation of left-right symmetry of $1 \mathrm{D}$ electron transport, characterized by a dispersion asymmetry parameter $\lambda_{a}$, appears. We show now that in quantum wires with broken chiral symmetry the spin-orbit interaction enhances a persistent current.

There is a close analogy between the Josephson current in a long SNS junction and the persistent current in a normal metal ring. For a long $\left(L>\hbar_{F} / \mid \Delta\right)$ SNS junction Andreev boundary conditions can be recast [22] in the form of twisted boundary conditions for chiral ( right- or left-moving) fermions on a ring with circumference $2 L$ pierced by the magnetic flux $\Phi / \Phi_{0}=1 / 2 \pm \varphi / 2 \pi$, where $\Phi_{0}=h c / e$ is the normal flux quantum. Due to this mapping the corresponding formulas for the persistent current in a normal diamagnetic (with odd number of spinless fermions) 1D ring and the formulas for the Josephson current coincide up to numerical factors. Here we consider the influence of dispersion asymmetry in the electron spectrum on the off-resonance supercurrent through a single barrier SNS junction (notice that resonace effects disappear in zero magnetic field).

In a low-transparency 1D SNS junction the critical current in the presence of dispersion asymmetry is of the form $J_{c}=f\left(\lambda_{a}\right) D e v_{F} / L$. To evaluate the function $f\left(\lambda_{a}\right)$ analytically we consider two limiting cases: (i) an asymmetric junction $l=0$, and (ii) a symmetric junction $l=L / 2$.

The first case models a junction with strong normal backscattering at one of the two interfaces. In zero magnetic field the spectral equation, Eq. (3), in the limit considered is reduced to

$$
\cos \left(E \delta_{L}^{(+)}\right) \simeq-\frac{D}{2} \cos \left(E \delta_{L}^{(-)}+\eta \varphi\right) .
$$

The energy spectrum and the partial supercurrents in the limit $D<<1$ are

$$
\begin{gathered}
E_{n}^{(0)}=\frac{\pi}{2 \delta_{L}^{(+)}}\left(n+\frac{1}{2}\right), \\
j_{n, \eta}^{(a)}=\eta(-1)^{n+1} \frac{e}{\hbar} \frac{D}{2 \delta_{L}^{(+)}} \sin \left[\pi\left(n+\frac{1}{2}\right) \lambda_{a}+\eta \varphi\right] .
\end{gathered}
$$

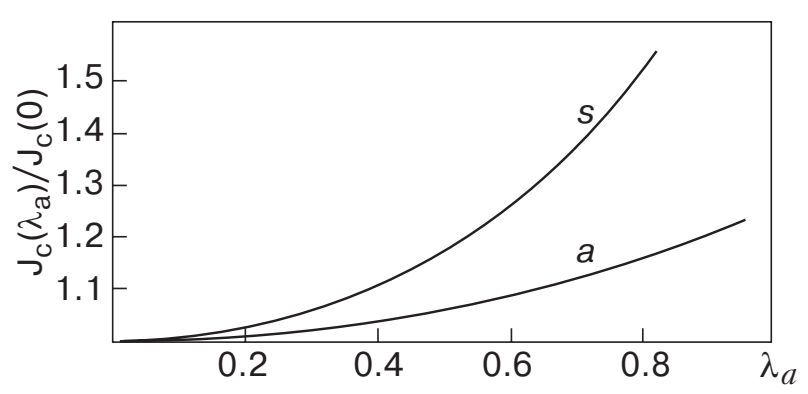

Fig. 4. The dependence of the normalized critical current $J_{c}\left(\lambda_{a}\right) / J_{c}\left(\lambda_{a}=0\right)$ in a low-transparency $(D<<1) \mathrm{S}-\mathrm{N}-\mathrm{S}$ junction on the parameter of dispersion asymmetry $\lambda_{a}$. The curve labelled by «s» corresponds to the case of symmetric junction $(l=L / 2)$, while the curve « $a »$ describes a strongly asymmetric junction $(l=0)$. 
By summing partial currents over quantum numbers of occupied states $\left(E_{n}^{(0)} \leq 0\right)$ at $T=0$ one gets

$$
\begin{gathered}
J^{(a)}=\sum_{n=-1 ; \eta= \pm 1}^{\infty} j_{n, \eta}^{(a)}=J_{c}^{(a)}\left(\lambda_{a}\right) \sin \varphi, \\
J_{c}^{(a)}\left(\lambda_{a}\right)=\frac{e v_{F}}{4 L} D \frac{1-\lambda_{a}^{2}}{\cos \left(\pi \lambda_{a} / 2\right)} .
\end{gathered}
$$

The critical current in the absence of spin-orbit interaction $J_{c}^{(a)}(0)=\operatorname{Dev}_{F} / 4 L$ coincides with the known results (see, e.g., Ref. 16). The normalized current $J_{c}^{(a)}(\varphi) / J_{c}^{(a)}(0)$ is shown in Fig. 4 (curve «a»).

For a symmetric junction the analogous calculation leads to the expression

$$
J_{c}^{(s)}\left(\lambda_{a}\right)=\frac{e v_{F}}{\pi L} D \frac{\pi \lambda_{a}\left(1-\lambda_{a}^{2}\right)}{\sin \left(\pi \lambda_{a}\right)} ; \quad J_{c}^{(s)}(0)=\frac{e v_{F}}{\pi L} D .
$$

The curve labelled by «s» in Fig. 4 demonstrates the dependence of the critical current in a symmetric junction on the spin-orbit parameter $\lambda_{a}$. We see that the spin-orbit Rashba interaction in quasi-1D quantum wires always enhances the critical current. The qualitative explanation of the unusual impact of chiral symmetry breaking on the critical Josephson current is as follows. The Josephson current (as any other thermodynamic persistent current) is a sum of partial currents of all occupied energy levels. The partial currents of adjacent energy levels are opposite in sign, and for chiral invariant systems (i.e., without dispersion asymmetry) they almost perfectly cancel each other to produce a net current of the order of a single-level current. When chiral invariance is broken the absolute values of partial currents of adjacent energy levels are different, and the concellation of currents for distant levels is less perfect. It results in an enhancement of the critical Josephson current. The effect is, however, numerically not large.

\section{Conclusion}

In quantum wires formed in a two-dimensional electron gas (2DEG) by lateral confinement the Rashba spin-orbit interaction is not reduced to a pure 1D Hamiltonian $\mathcal{H}_{1 D}^{(s o)}=\alpha_{s o} \hat{p}_{x} \sigma_{z}$. As was shown in Ref. 8 the presence of an in-plane confinement potential qualitatively modifies the energy spectrum of the 1D electrons so that a dispersion asymmetry appears. As a result the chiral symmetry is broken in quantum wires with Rashba coupling. Although the effect was shown [8] not to be numerically large, the breakdown of symmetry leads to qualitatively novel predictions.
We have considered here the influence of dispersion asymmetry and Zeeman splitting on the Josephson current through a superconductor/quantum wire/superconductor junction. We showed that the violation of chiral symmetry in a quantum wire results in qualitatively new effects in a weak superconductivity. In particularly, the interplay of Zeeman and Rashba interactions induces a Josephson current through the hybrid 1D structure even in the absence of any phase difference between the superconductors. At low temperatures $\left(T<<\hbar v_{F} / L\right)$ the anomalous supercurrent can be of the order of the critical Josephson current. For a transparent junction with small or moderate dispersion asymmetry (characterized by the dimensionless parameter $\left.\lambda_{a}=\left(v_{1 F}-v_{2 F}\right) /\left(v_{1 F}+v_{2 F}\right)\right)$ it appears, as a function of the Zeeman splitting $\Delta_{Z}$, abruptly at $\Delta_{Z} \sim \hbar v_{F} / L$. In a low-transparency $(D<<1)$ junction, the anomalous Josephson current under special (resonance) conditions is of the order of $\sqrt{D}$. In zero magnetic field the anomalous supercurrent disappears (as it should) since the spin-orbit interaction itself respects T-symmetry. However, the influence of the spin-orbit interaction on the critical Josephson current through a quasi-1D structure is still anomalous. Contrary to what holds for chiral invariant systems with the Aharonov-Bohm geometry, where spin-orbit effects suppress persistent currents [15], the breakdown of chiral symmetry results in an enhancement of the supercurrent.

All the phenomena described above are absent in a 2D-junction when the effects of transverse mode quantization are neglected [11]. We have considered the limiting case of a single (transverse) channel because this is the case for which the effects induced by a dispersion asymmetry in the electron spectrum are most pronounced. The anomalous supercurrent Eq. (7) is a sign-alternating function of the transverse channel index, since for neighboring channels the spin projections of the chiral states are opposite [8]. Besides, the absolute value of the dispersion asymmetry parameter $\lambda_{a}^{(j)}$ decreases with transverse-channel number $j$. So, for a multichannel junction the effects related to a dispersion asymmetry phenomenon will be strongly suppressed, and they completely disappear in the pure 2D case.

We evaluated the Josephson current through a $\mathrm{S}-\mathrm{QW}-\mathrm{S}$ junction in a model of noninteracting electrons. In QWs the effects of electron-electron interaction can be significant, and here we comment on how interaction effects could modify the obtained results. Electronic properties of $1 \mathrm{D}$ QWs are usually described by a Luttinger liquid ( LL ) model (see, e.g., Ref. 23). The supercurrent in a S-LL-S junction with repulsive electron-electron interaction strongly de- 
pends on the quality of electric contact of a nanowire with the bulk superconductors. For adiabatic contacts, when only Andreev scattering takes place at the interfaces, the Josephson current through a perfect wire is not renormalized by interaction [22,24]. For tunnel contacts electron-electron interaction in a wire renormalizes the barrier transparencies (Kane-Fisher effect [25]), and for repulsive interaction the critical current is strongly suppressed [26].

We are interested in spin-orbit effects in a $\mathrm{S}-\mathrm{QW}-\mathrm{S}$ junction. It is reasonably to expect that Eqs.(6),(7) derived for a perfect junction (without normal backscattering) will be valid even for interacting electrons. One could expect also that in a tunneling regime $(D<<1)$ the interaction effects in Eqs. (13),(17),(18) can be estimated by replacing the bare transparency $D$ by the interaction-renormalized one [22] $D_{g} \sim D(d / L)^{K_{\rho}^{-1}-1}$ (here $d \ll L$ is the width of the wire and $K_{\rho}$ is the LL correlation parameter). In quantum wires formed in 2DEG the Coulomb interaction is not strong [1] and it can be controlled by the gate electrodes. For weakly interacting electrons (a conceivable case for «semiconducting» QWs), when the electron picture of charge transport through a $1 \mathrm{D}$ wire is still valid [27], the interaction effects can actually be accounted for by replacing the bare transmission coefficient by the renormalized one.

The authors thank E. Bezuglyi, A. Kadigrobov, and V. Shumeiko for fruitful discussions. This research is supported by the Royal Swedish Academy of Sciences (KVA) and by the Swedish Research Council (LYG, RIS). IVK acknowledges the hospitality of Department of Applied Physics, Chalmers University of Technology and Göteborg University.

\section{Appendix}

We consider electron transport through a normal region of length $L$ with a local scatterer placed a distance $l$ from the left bank, at point $x=l$. In our two-channel chiral model (the term «chiral» here means that left- and right-moving particles with a given Fermi velocity have opposite spin polarizations; see Fig. 1) backscattering by a nonmagnetic impurity corresponds to a backward interchannel scattering ( $\ll 1 » \Leftrightarrow\langle 2 »)$. In the presence of a magnetic field and interchannel scattering the general $4 \times 4$ nonsymmetric $\mathrm{S}$-matrix can be parametrized as follows

$\hat{S}_{E}=\left(\begin{array}{ll}|r| \sigma_{1} \exp i\left(\rho_{R}+\sigma_{3} \mu_{R}\right) & |t| \sigma_{3} \exp i\left(\alpha_{R}+\sigma_{3} \eta_{R}\right) \\ |t| \sigma_{3} \exp i\left(\alpha_{L}+\sigma_{3} \eta_{L}\right) & |r| \sigma_{1} \exp i\left(\rho_{L}+\sigma_{3} \mu_{L}\right)\end{array}\right)$.
Here $\sigma_{1,3}$ are the Pauli matrices, $t_{R, L}=|t| \exp \left(i \eta_{R, L}\right)$; $r_{R, L}=|r| \exp \left(i_{\rho_{R, L}}\right)$ and $|t|^{2}+|r|^{2}=1$. Unitarity of the S-matrix $\left(S S^{\dagger}=I\right)$ implies the relations

$$
\mu_{R}+\eta_{R}=\mu_{L}+\eta_{L}, \rho_{R}+\rho_{L}=\alpha_{R}+\alpha_{L} .
$$

Thus, the scattering matrix in our problem is described by 7 independent real parameters. For a point scatterer with energy-independent scattering amplitudes $\left(t_{0}, r_{0}\right)$ one readily gets that $|t|^{2}=\left|t_{0}\right|^{2} \equiv D$ ( $D$ is the junction transparency). The phases are

$$
\begin{gathered}
\mu_{R}=-(L-l) k_{-}, \rho_{R}=(L-l) k_{+}, \eta_{R}=L k_{-}^{\uparrow \downarrow} \\
\alpha_{R}=L k_{+}^{\uparrow \downarrow}, \mu_{L}=l k_{-}, \rho_{L}=l k_{+}, \eta_{L}=L k_{-}^{\downarrow \uparrow}, \\
\alpha_{L}=L k_{+}^{\downarrow \uparrow},
\end{gathered}
$$

where

$$
\begin{gathered}
k_{ \pm}=k_{+}^{\uparrow \uparrow} \pm k_{+}^{\downarrow \downarrow}, \quad k_{ \pm}^{\sigma \sigma^{\prime}}=\frac{1}{2}\left(k_{1}^{\sigma^{\prime}} \pm k_{2}^{\sigma^{\prime}}\right), \\
k_{j}^{\sigma}( \pm E)=k_{j F}+\frac{ \pm E+\sigma g \mu_{B} H / 2}{\hbar v_{j F}}
\end{gathered}
$$

and $k_{j}, v_{j F}(j=1,2)$ are the Fermi momentum and the Fermi velocity in the $j$ th channel.

The diagonal matrix $\hat{r}_{A}$ in Eq. (2) takes the form

$$
\hat{r}_{A}=\left(\begin{array}{cc}
\exp (i \varphi / 2 i) \hat{I} & 0 \\
0 & \exp (-i \varphi / 2 i) \hat{I}
\end{array}\right)
$$

where $\varphi$ is the superconducting phase difference and $\hat{I}$ is the $2 \times 2$ unit matrix.

1. S. Tarucha, T. Honda, and T. Saku, Solid State Commun. 94, 413 (1995); A. Yakoby, H.L. Stormer, N.S. Wigreen, L.N. Pfeiffer, R.W. Baldwin, and K.W. West, Phys. Rev. Lett. 77, 4612 (1996).

2. S.J. Tans, M.H. Devoret, H. Dai, A. Thess, R.E. Smalley, L.J. Geerlings, and C. Dekker, Nature 386, 474 (1997).

3. R. Egger and A.O. Gogolin, Phys. Rev. Lett. 79, 5082 (1997); C.L. Kane, L. Balents, and M.P.A. Fisher, Phys. Rev. Lett. 79, 5086 (1997).

4. M. Bockrath, D.H. Cobden, J. Lu, A.G. Rinzler, G. Andrew, R.E. Smalley, L. Balents, and P.L. McEuen, Nature 397, 598 (1999); Z. Yao, H.W.J. Postma, L. Balents, and C. Dekker, Nature 402, 273 (1999).

5. E.I. Rashba, Fiz. Tverd. Tela (Leningrad) 2, 1224 (1960) [Sov. Phys. Solid. State 2, 1109 (1960)]; Y.A. Bychkov and E.I. Rashba, J. Phys. C17, 6039 (1984).

6. J. Nitta, T. Akasaki, H. Takayanagi, and T. Enoki, Phys. Rev. Lett. 78, 1335 (1997); D. Grundler, Phys. Rev. Lett. 84, 6074 (2000).

7. F. Mireles and G. Kirczenow, Phys. Rev. B64, 024426 (2001).

8. A.V. Moroz and C.H. Barnes, Phys. Rev. B60, 14272 (1999). 
9. A.V. Moroz, K.V. Samokhin, and C.H. Barnes, Phys. Rev. B62, 16900 (2000).

10. M. Governale and U. Zulicke, Phys. Rev. B66, 073311 (2002).

11. E.V. Bezuglyi, A.S. Rozhavsky, I.D. Vagner, and P. Wyder, Phys. Rev. B66, 052508 (2002).

12. P.G. de Gennes and D. Saint-James, Phys. Lett. 4, 151 (1963).

13. G. Wendin and V.S. Shumeiko, Phys. Rev. B53, R6006 (1996), Superlattices Microstruct. 4, 569 (1996).

14. P. Sandström and I.V. Krive, Phys. Rev. B56, 9255 (1997).

15. Y. Meir, Y. Gefen, and O. Entin-Wohlman, Phys. Rev. Lett. 63, 798 (1989).

16. P. Samuelsson, J. Lantz, V.S. Shumeiko, and G. Wendin, Phys. Rev. B62, 1319 (2000).

17. A. Svidzinsky, T.N. Antsygina, and E.N. Bratus', ZhETF 61, 1612 (1971) [Sov. Phys. JETP 34, 860 (1972)].
18. V.M. Mostepanenko and N.N. Trunov, The Casimir Effect and its Application, Claredon Press, Oxford (1997).

19. C.W.J. Beenakker, Phys. Rev. Lett. 67, 3836 (1991).

20. P.F. Bagwell, Phys. Rev. B46, 12573 (1992).

21. I.O. Kulik, Zh. Eksp. Teor. Fiz. 57, 1745 (1969) [Sov. Phys. JETP 30, 944 (1970)].

22. D.L. Maslov, M. Stone, P.M. Goldbart, and D. Loss, Phys. Rev. B53, 1548 (1996).

23. A.O. Gogolin, A.A. Nersesyan, and A.M. Tsvelik, Bosonization and Strongly Correlated Systems, Cambridge University Press (1998).

24. Affleck, J.-S. Caux, and A. Zagoskin, Phys. Rev. B62, 1433 (2000).

25. C.L. Kane and M.P.A. Fisher, Phys. Rev. Lett. 68, 1220 (1992); Phys. Rev. B46, 15233 (1992).

26. R. Fazio, F.W.J. Hekking, and A.A. Odintsov, Phys. Rev. Lett. 74, 1843 (1995).

27. K.A. Matveev, D. Yue, and L.I. Glazman, Phys. Rev. Lett. 71, 3351 (1993). 